\title{
Hall effects on MHD Oscillatory flow of Non-Newtonian Fluid through porous medium in a Vertical channel with suction/injection
}

\author{
M.Kalpana ${ }^{1 *}$ and R.Bhuvana Vijaya ${ }^{2}$ \\ ${ }^{1}$ Research Scholar, Dept. of Mathematics, JNTUA College of Engineering, Ananthapuramu, Andhra Pradesh - 515002, India.
}

${ }^{2}$ Professor, Dept. of Mathematics, JNTUA College of Engineering, Ananthapuramu, Andhra Pradesh - 515002, India.

\begin{abstract}
In this paper, we investigate the effect of suction/injection on the unsteady MHD oscillatory second grade fluid flow through a vertical channel with non-uniform wall temperature. The fluid is subjected to Hall effects and the velocity slip at the lower plate is taken into consideration. Exact solutions of the dimensionless equations governing the fluid flow are obtained and the effects of the flow parameters on temperature, velocity profiles, skin friction and rate of heat transfer are discussed and shown graphically. It is interesting to note that skin friction increases on both channel plates as injection increases on the heated plate.
\end{abstract}

Keywords: Hall effects, Oscillatory flow, Porous medium, Magnetic field, Fluid slip, Suction/injection

\section{INTRODUCTION}

The study of oscillatory flow of an electrically conducting fluid through a porous channel saturated with porous medium is important in many physiological flows and engineering applications such as magnetohydrodynamic (MHD) generators, arterial blood flow, petroleum engineering. Makinde and Mhone [1] investigated the forced convective MHD oscillatory fluid flow through a channel filled with porous medium, and analyses were based on the assumption that the plates are impervious. In a related study, Mehmood and Ali [2] investigated the effect of slip on the free convective oscillatory flow through vertical channel with periodic temperature and dissipative heat. In addition, Chauchan and Kumar [3] studied the steady flow and heat transfer in a composite vertical channel. Palani and Abbas [4] investigated the combined effects of magneto-hydrodynamics and radiation effect on free convection flow past an impulsively started isothermal vertical plate using the Rossel and approximation. Hussain et al. [5] presented analytical study of oscillatory second grade fluid flow in the presence of a transverse magnetic field. Umavathi et al. [6] investigated the unsteady flow of viscous fluid through a horizontal composite channel. Ajibade and Jha [7] presented the effects of suction and injection on hydrodynamics of oscillatory fluid through parallel plates. Ajibade and Jha [8] extended the problem to heat generating/absorbing fluids, the effect of viscous dissipation of the free convective flow with time dependent boundary condition was investigated (Ajibade and Jha [9]). More recently, Adesanya and Makinde [10] discussed the radiative heat transfer on the pulsatile couple stress fluid flow with time dependent boundary condition. Adesanya and Gbadeyan [11] studied the flow and heat transfer of steady non-Newtonian fluid flow noting the fluid slip in the porous channel. Many authors [12-20] discussed some problems on MHD flows in different physical configurations. Veera Krishna et al. [21] discussed heat and mass transfer on unsteady MHD oscillatory flow of blood through porous arteriole. The effects of radiation and Hall current on MHD flow through porous medium have studied by Veera Krishna et al. [22]. The heat generation/absorption and thermo-diffusion on MHD flow of radiating and chemically reactive second grade fluid near an infinite vertical plate through a porous medium with Hall effect have studied by Veera Krishna and Chamkha [23]. Veera Krishna and Chamkha [25] investigated The diffusion-thermo, radiationabsorption and Hall and ion slip effects on MHD free convective rotating flow of nano-fluids $(\mathrm{Ag}$ and $\mathrm{TiO} 2)$ past a semi-infinite permeable moving plate with constant heat source. Veera Krishna et al.[26] discussed the Soret and Joule effects of MHD mixed convective flow of an incompressible and electrically conducting viscous fluid past an infinite vertical porous plate taking Hall effects into account. Veera Krishna and Chamkha [27] discussed the MHD squeezing flow of a water-based nanofluid through a saturated porous medium between two parallel disks, taking the Hall current into account.

Motivated by the above studies, we investigate the effect of suction/injection on the unsteady oscillatory second grade fluid flow through a vertical channel with non-uniform wall temperature and taking Hall current into account.

\section{FORMULATION AND SOLUTION OF THE PROBLEM}

We consider mass transfer on the unsteady laminar flow of an incompressible viscous electrically conducting second grade fluid through a channel with slip at the cold plate. An external magnetic field is placed across the normal to the channel and taking Hall current into account. It is assumed that the fluid has small electrical conductivity and the electro-magnetic force produced is also very small. The flow is subjected to suction at the cold wall and injection at the heated wall. We choose a Cartesian coordinate system $(x, y, z)$ where $x$ lies along the centre of the channel, and $z$ is the distance measured 
in the normal section such that $\mathrm{z}=d$ is the channel's half width as shown in the Fig.1.

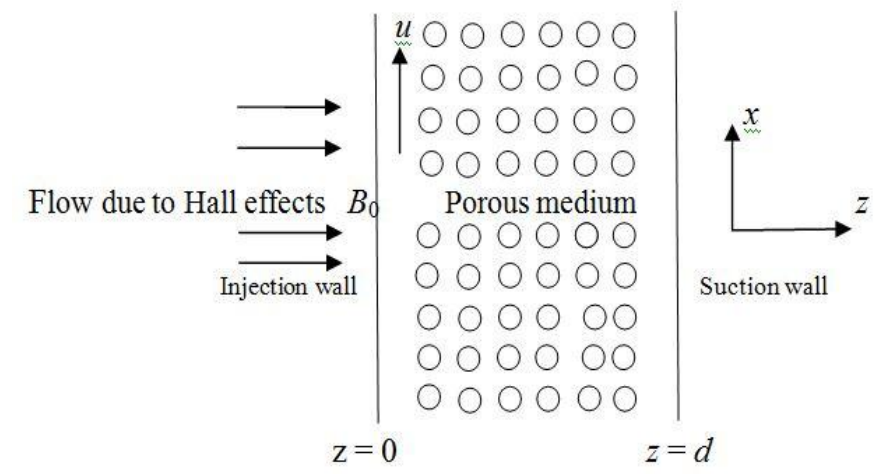

Fig. 1 Physical Configuration of the Problem

Under the usual Bousinesq approximation the equations governing the flow are as follows:

Equation of continuity:

$\frac{\partial u}{\partial x}+\frac{\partial v}{\partial y}=0$

Momentum of equations

$$
\begin{aligned}
\frac{\partial u}{\partial t}-w_{0} \frac{\partial u}{\partial z} & =-\frac{1}{\rho} \frac{\partial p}{\partial x}+v \frac{\partial^{2} u}{\partial z^{2}}+\frac{\alpha_{1}}{\rho} \frac{\partial^{3} u}{\partial z^{2} \partial t}+B_{0} J_{y} \\
& -\frac{v}{K_{1}} u+g \beta\left(T-T_{0}\right)+g \beta^{*}\left(C-C_{0}\right)
\end{aligned}
$$

$$
\begin{aligned}
\frac{\partial v}{\partial t} & -w_{0} \frac{\partial v}{\partial z} \\
& =-\frac{1}{\rho} \frac{\partial p}{\partial y}+v \frac{\partial^{2} v}{\partial z^{2}}+\frac{\alpha_{1}}{\rho} \frac{\partial^{3} v}{\partial z^{2} \partial t}-B_{0} J_{x}-\frac{v}{K_{1}} v
\end{aligned}
$$

Equation of energy

$$
\frac{\partial T}{\partial t}-w_{0} \frac{\partial T}{\partial z}=\frac{k_{f}}{\rho C_{p}} \frac{\partial^{2} T}{\partial z^{2}}+\frac{4 \alpha^{2}}{\rho C_{p}}\left(T-T_{0}\right)
$$

Equation of concentration

$$
\frac{\partial C}{\partial t}-w_{0} \frac{\partial C}{\partial z}=D \frac{\partial^{2} C}{\partial z^{2}}-k_{1}\left(C-C_{0}\right)
$$

Where, all the physical quantities are their usual meaning. When the strength of the magnetic field is very large, the generalized Ohm's law is modified to include the Hall current so that

$$
\boldsymbol{J}+\frac{\omega_{e} \tau_{e}}{B_{0}}(\boldsymbol{J} \times \boldsymbol{B})=\sigma\left[\boldsymbol{E}+\boldsymbol{V} \times \boldsymbol{B}+\frac{1}{e \eta_{e}} \nabla P_{e}\right\rfloor
$$

The ion-slip and thermo electric effects are not included. Further it is assumed that $\omega_{e} \tau_{e} \sim 0$ (1) and $\omega_{i} \tau_{i}<<1$, where $\omega_{i}$ and $\tau_{i}$ are the cyclotron frequency and collision time for ions respectively. In the equation (6) the electron pressure gradient, the ion-slip and thermo-electric effects are neglected. We also assume that the electric field $\boldsymbol{E}=0$ under assumptions reduces to

$J_{x}+m J_{y}=\sigma B_{0} v$

$J_{y}-m J_{x}=-\sigma B_{0} u$

On solving equations (7) and (8) we obtain,

$J_{x}=\frac{\sigma B_{0}}{1+m^{2}}(v+m u)$

$J_{y}=\frac{\sigma B_{0}}{1+m^{2}}(m v-u)$

Substituting the equations (9) and (10) in (3) and (2) respectively, we obtain

$$
\begin{aligned}
\frac{\partial u}{\partial t}-w_{0} \frac{\partial u}{\partial z}= & -\frac{1}{\rho} \frac{\partial p}{\partial x}+v \frac{\partial^{2} u}{\partial z^{2}}+\frac{\alpha_{1}}{\rho} \frac{\partial^{3} u}{\partial z^{2} \partial t}+\frac{\sigma B_{0}^{2}}{1+m^{2}}(m v-u) \\
& -\frac{v}{K_{1}} u+g \beta\left(T-T_{0}\right)+g \beta^{*}\left(C-C_{0}\right)
\end{aligned}
$$

$$
\begin{aligned}
& \frac{\partial v}{\partial t}-w_{0} \frac{\partial v}{\partial z}=-\frac{1}{\rho} \frac{\partial p}{\partial y}+v \frac{\partial^{2} v}{\partial z^{2}} \\
& +\frac{\alpha_{1}}{\rho} \frac{\partial^{3} v}{\partial z^{2} \partial t}-\frac{\sigma B_{0}^{2}}{1+m^{2}}(v+m u)-\frac{v}{K_{1}} v
\end{aligned}
$$

Combining Eqns. (11) and (12), Let $q=u+i v$ and $\xi=x-i y$, we obtain

$$
\begin{aligned}
\frac{\partial q}{\partial t}- & w_{0} \frac{\partial q}{\partial z}=-\frac{1}{\rho} \frac{\partial p}{\partial \xi}+v \frac{\partial^{2} q}{\partial z^{2}}+\frac{\alpha_{1}}{\rho} \frac{\partial^{3} q}{\partial z^{2} \partial t} \\
& -\frac{\sigma B_{0}^{2}}{\rho(1-i m)} q-\frac{v}{K_{1}} q+g \beta\left(T-T_{0}\right)+g \beta^{*}\left(C-C_{0}\right)
\end{aligned}
$$

The boundary conditions are

$q=\frac{\sqrt{k}}{\alpha_{s}} \frac{\partial q}{\partial z}, T=T_{0}, C=C_{0} \quad$ at $z=0$

$q=0, T=T_{1}, C=C_{1} \quad$ at $\quad z=d$ 
Introducing the dimensionless parameters variables are given as

$$
\begin{aligned}
& \left(x^{*}, z^{*}\right)=\frac{(x, z)}{d}, q=\frac{q^{*} v}{d}, t=\frac{t^{*} d^{2}}{v}, \\
& p=\frac{p^{*} \rho v^{2}}{d^{2}}, \theta=\frac{T-T_{0}}{T_{1}-T_{0}}, \phi=\frac{C-C_{0}}{C_{1}-C_{0}}, \\
& \mathrm{Gr}=\frac{\mathrm{g} \beta\left(T_{1}-T_{0}\right) d^{3}}{v^{2}}, \mathrm{Gm}=\frac{g \beta\left(C-C_{0}\right) d^{3}}{v^{2}}, \\
& \operatorname{Pr}=\frac{\rho c_{p} v}{k}, \delta=\frac{4 \alpha^{2} d^{2}}{\rho c_{p} v}, \gamma=\frac{\sqrt{k}}{\alpha_{s} d}, \\
& M^{2}=\frac{\sigma B_{0}^{2} d^{2}}{\rho v}, K=\frac{K_{1}}{d^{2}}, s=\frac{\omega_{0} d}{\gamma}, \\
& x^{*}=\frac{x}{d}, y^{*}=\frac{y}{d}, z^{*}=\frac{z}{d}
\end{aligned}
$$

Making use of non-dimensional variables, we obtain the dimensionless governing equations (Dropping asterisks) and are

$$
\begin{aligned}
\frac{\partial q}{\partial t}- & s \frac{\partial q}{\partial z}=-\frac{\partial p}{\partial \xi}+\frac{\partial^{2} q}{\partial z^{2}}+\alpha \frac{\partial^{3} q}{\partial z^{2} \partial t} \\
& -\left(\frac{M^{2}}{1-i m}+\frac{1}{K}\right) q+\operatorname{Gr} \theta+G m \phi
\end{aligned}
$$

$$
\begin{aligned}
& \frac{\partial \theta}{\partial t}-s \frac{\partial \theta}{\partial z}=\frac{1}{\operatorname{Pr}} \frac{\partial^{2} \theta}{\partial z^{2}}+\delta \theta \\
& \frac{\partial \phi}{\partial t}-s \frac{\partial \phi}{\partial z}=\frac{1}{\operatorname{Sc}} \frac{\partial^{2} \phi}{\partial z^{2}}-\operatorname{KcSc} \phi
\end{aligned}
$$

With the appropriate boundary conditions

$$
\begin{aligned}
& q=\gamma \frac{\partial q}{\partial z}, \theta=0, \phi=0 \text { at } z=0 \\
& q=0, \theta=0, \phi=1 \text { at } z=1
\end{aligned}
$$

We assume that an oscillatory pressure gradient such that solution of the dimensionless equations (16) - (18) is in the following form:

$$
\begin{gathered}
-\frac{d p}{d \xi}=P e^{i \omega t}, q(z, t)=q_{0}(z) \mathrm{e}^{i \omega t}, \\
\theta(z, t)=\theta_{0}(z) \mathrm{e}^{i \omega t}, \phi(z, t)=\phi_{0}(z) \mathrm{e}^{i \omega t}
\end{gathered}
$$

In view of (21) equations (10) - (14) reduced to a boundary value-problem in the following form.

$$
\begin{aligned}
& (1+\alpha \mathrm{i} \omega) \frac{d^{2} q_{0}}{d z^{2}}+s \frac{d q_{0}}{d z}-\left(\frac{M^{2}}{1-i m}+\frac{1}{K}+\mathrm{i} \omega\right) q_{0} \\
& =-P+\mathrm{Gr} \theta_{0}+\mathrm{Gm} \phi_{0} \\
& \frac{d^{2} \theta_{0}}{d z^{2}}+s \operatorname{Pr} \frac{d \theta_{0}}{d z}+(\delta-\mathrm{i} \omega) \operatorname{Pr} \theta_{0}=0 \\
& \frac{d^{2} \phi_{0}}{d z^{2}}+s \mathrm{Sc} \frac{d \phi_{0}}{d z}+(\mathrm{KcSc}-\mathrm{i} \omega) \operatorname{Sc} \phi_{0}=0
\end{aligned}
$$

Corresponding boundary conditions are

$$
\begin{aligned}
& q_{0}(0)=\gamma \frac{d q_{0}}{d z}, q_{0}(1)=0, \theta_{0}(0)=0, \\
& \theta_{0}(1)=1, \phi_{0}(0)=0, \phi_{0}(1)=1
\end{aligned}
$$

The solutions of the (22) to (24) with respect to boundary conditions (25), we obtain the velocity, temperature and concentration.

For engineering intrest, the shear stress, the rate of heat transfer and rate of mass transfer is given by

$$
\tau=\frac{\partial q}{\partial z}, N u=\frac{\partial \theta}{\partial z} \text { and } S h=\frac{\partial \phi}{\partial z}
$$

\section{RESULTS AND DISCUSSION}

The Figs. (2-14), Figs. (15) and Figs. (16) represent the velocity profiles, temperature profiles and concentration profiles respectively. Tables (1-3) are shown Shear stress, Nuselt number $(\mathrm{Nu})$, and Sherwood number (Sh). We have drawn the all profiles with respect to the different variation in the governing parameters being the other parameter fixed. $P=1, t=0.1$.

We noticed from the Figs. 2 and 3 that the magnitude of the velocity components $u$ and $v$ increases with increasing $m$ and $\alpha$. In Figs. 4, the effect of the retarding effect of Lorentz forces present in the magnetic field on the fluid flow is presented. It is observed that maximum flow occurs in the absence of the magnetic field, and further increase in the Hartmann's number is seen to decrease the fluid velocity components $u$ and $v$. Figs. 5 present the plot of increase in channel porous permeability on the velocity profile. As observed, as the permeability of the medium increases there is increase in the fluid velocity since barriers placed on the flow path reduce as $K$ increases allowing for free flow thus increasing the velocity. 
The effect of the suction/injection parameter, Pr, the radiation parameter and the $\omega$ on the temperature of the fluid within the channel is shown in Figs. 6. From the plot, it is observed that fluid temperature is linearly distributed within the channel in the absence of suction/injection. However, as injection increases on the heated plate, fluid temperature increases within the channel. The concavity with increase in the suction/injection parameter is as a result of the direction of heat flow from the heated plate towards the cold plate. The magnitude of the temperature decreases with increasing Pr. Likewise as the radiation parameter increases, and the fluid temperature is seen to be increasing. This is due to the heat transfer from the heated wall to the fluid since the fluid absorbs its own radiations. An increase in the frequency of oscillation decreases the fluid temperature within the channel. This is attributed to a reduction in the heat transfer rate as the heating frequency.

We noticed that, from the Figures 7, the concentration increases with increasing suction parameter $s$, Schmidt number $S c$, or chemical reaction parameter $K c$ whereas it reduces with increasing the frequency of oscillation $\omega$.

Finally, Table 1 shows that the skin friction at both the walls. The stress components $\tau_{L}$ and $\tau_{R}$ increase with increasing
$\mathrm{K}, \mathrm{Gr}, \mathrm{Gm}$ and $s$ and reduces with increasing $M, \mathrm{Sc}, \mathrm{Kc}, \gamma$ and $\delta$. The stress components $\tau_{L}$ enhances and $\tau_{R}$ retards with $m, \alpha, \operatorname{Pr}$ and $\omega$. Table 2 shows the plot of heat transfer rate across the channel, and as observed that, the rate of heat transfer $\mathrm{Nu}_{\mathrm{L}}$ enhances and $\mathrm{Nu}_{\mathrm{R}}$ decreases with increasing $\operatorname{Pr}, s, \delta$ and $\omega$ in the fluid layer closer to the heated wall while it increases at the region close to the cold wall. The reason is that, heat is transferred from the heated plate to the fluid and from the fluid to the cold plate. Both $\mathrm{Nu}_{\mathrm{L}}$ and $\mathrm{Nu}_{\mathrm{R}}$ reduces with increasing time. Table 3 shows the mass transfer rate across the channel, and as observed that, the rate of mass transfer increases in the fluid layer closer to the heated wall while it decreases at the region close to the cold wall with increasing Schmidt number Sc, chemical reaction parameter Kc and Suction parameter $s$. Both $\mathrm{Sh}_{\mathrm{L}}$ and $\mathrm{Sh}_{\mathrm{R}}$ are reduces with increasing the frequency of oscillation and time $t$. Table 4 represents the comparison of the results for velocity component $u$ where $\quad \alpha \rightarrow 0, m \rightarrow 0 \quad$ with $\mathrm{Gm}=5, s=1, \operatorname{Pr}=0.71, \mathrm{Sc}=0.22$, $\mathrm{Kc}=1, \gamma=0.25, \omega=\pi / 6, \delta=0.5$. The results are good agreement with Falade et al. [24].

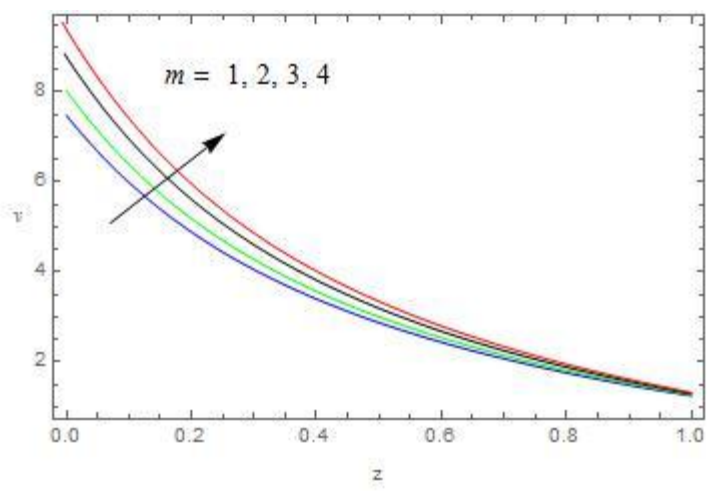

Figs. 2 The velocity profiles against $m$ with $M=0.5, \alpha=1, K=1, \mathrm{Gr}=4, \mathrm{Gm}=5, s=1, \mathrm{Pr}=0.71, \mathrm{Sc}=0.22, \mathrm{Kc}=1, \gamma=0.25, \omega=\pi / 6, \delta=0.5$
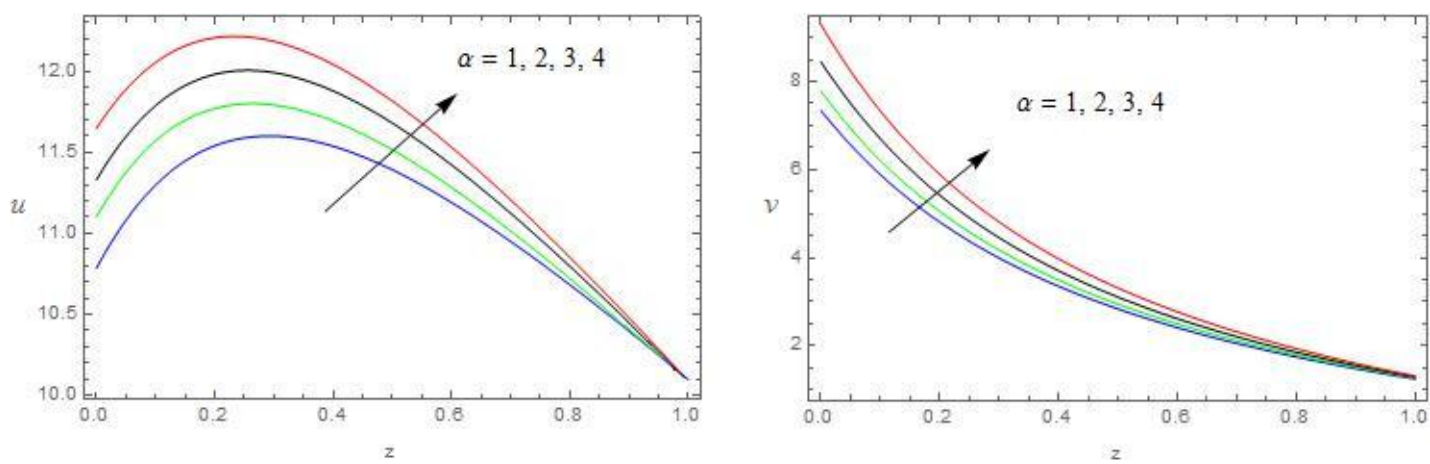

Figs. 3 The velocity profiles against $\alpha$ with $m=1, M=0.5, K=1, \mathrm{Gr}=4, \mathrm{Gm}=5, s=1, \mathrm{Pr}=0.71, \mathrm{Sc}=0.22, \mathrm{Kc}=1, \gamma=0.25, \omega=\pi / 6, \delta=0.5$ 
International Journal of Applied Engineering Research ISSN 0973-4562 Volume 14, Number 21 (2019) pp. $3960-3967$

(C) Research India Publications. https://dx.doi.org/10.37622/IJAER/14.21.2019.3960-3967
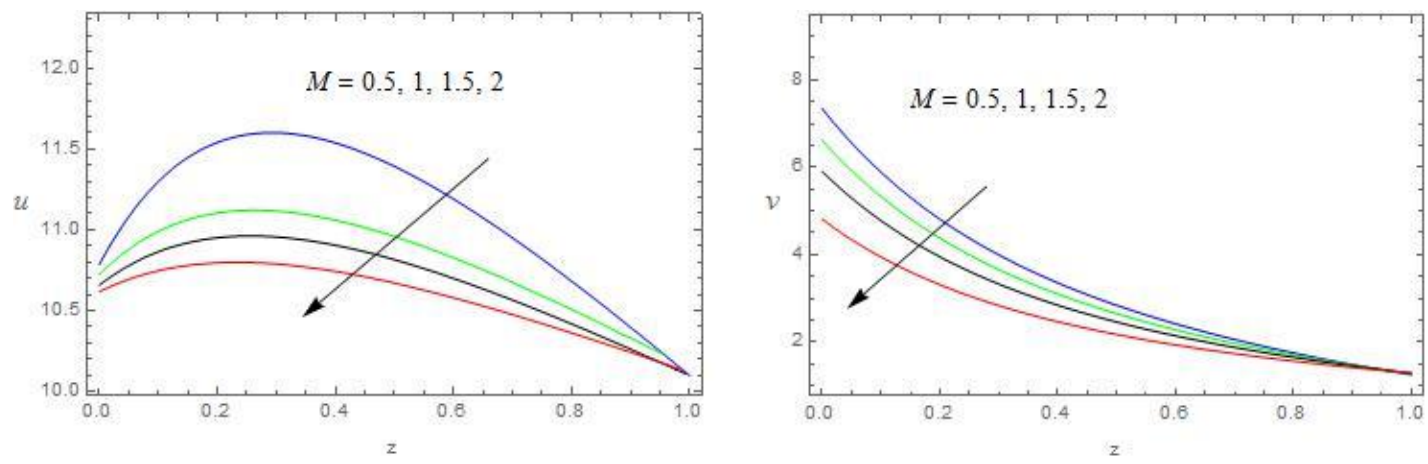

Figs. 4 The velocity profiles against $M$ with

$m=1, \alpha=1, K=1, \mathrm{Gr}=4, \mathrm{Gm}=5, s=1, \mathrm{Pr}=0.71, \mathrm{Sc}=0.22, \mathrm{Kc}=1, \gamma=0.25, \omega=\pi / 6, \delta=0.5$
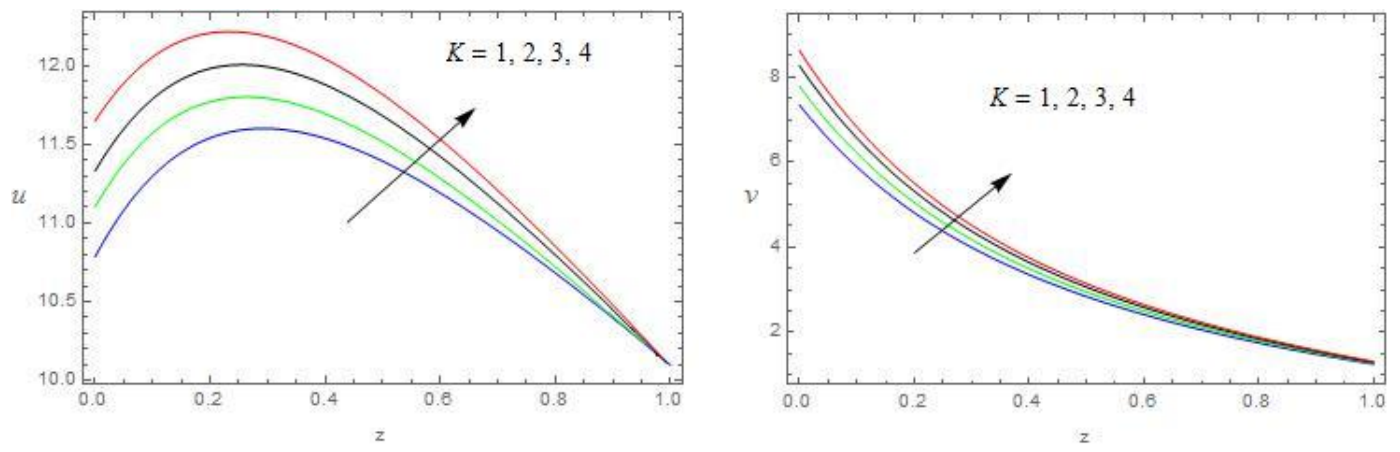

Figs. 5 The velocity profiles against $K$ with

$m=1, \alpha=1, M=0.5, \mathrm{Gr}=4, \mathrm{Gm}=5, s=1, \mathrm{Pr}=0.71, \mathrm{Sc}=0.22, \mathrm{Kc}=1, \gamma=0.25, \omega=\pi / 6, \delta=0.5$
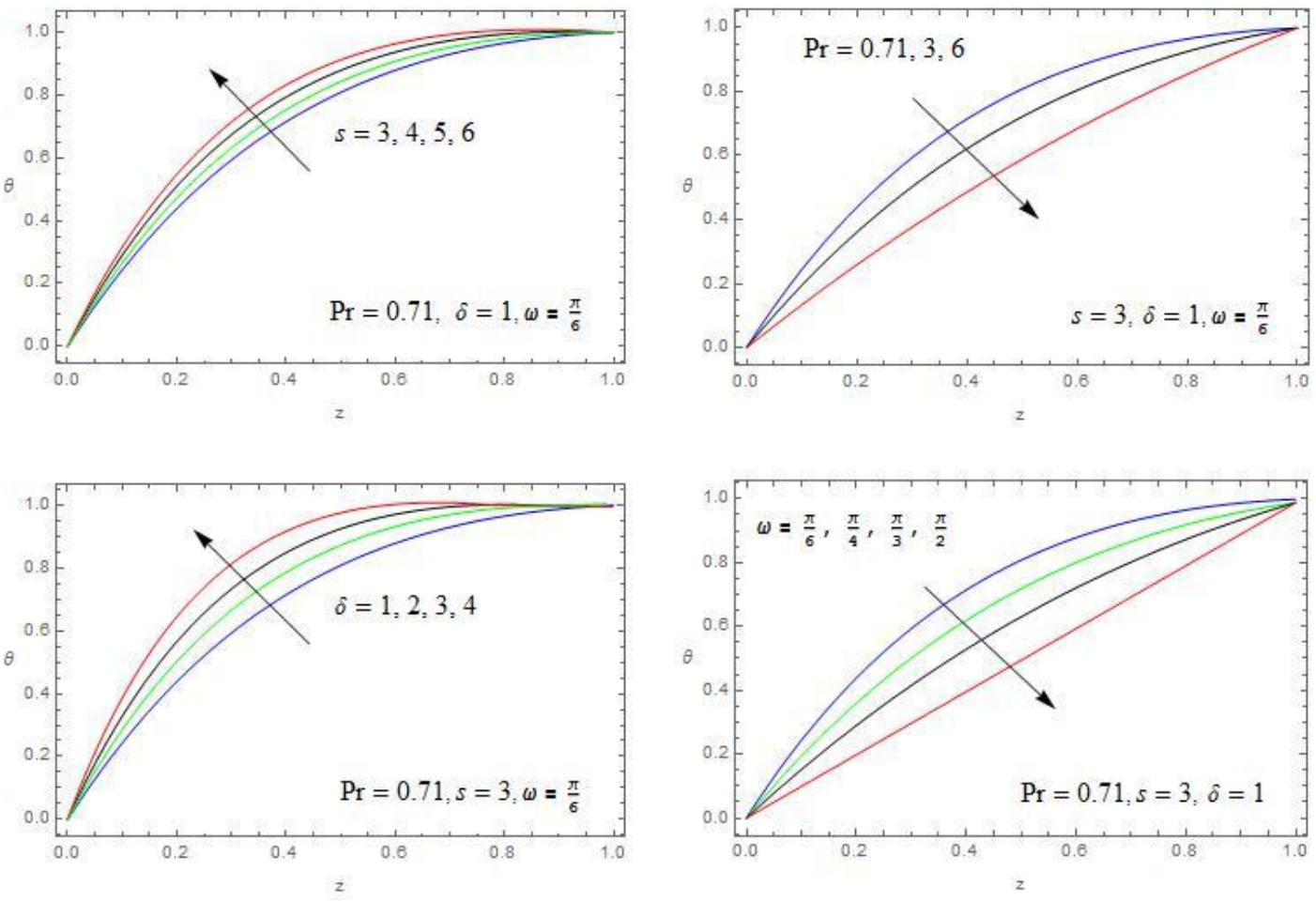

Fig 6: The Temperature profiles against $\mathrm{s}, \operatorname{Pr}, \delta, \omega$ 
International Journal of Applied Engineering Research ISSN 0973-4562 Volume 14, Number 21 (2019) pp. 3960-3967

(C) Research India Publications. https://dx.doi.org/10.37622/IJAER/14.21.2019.3960-3967
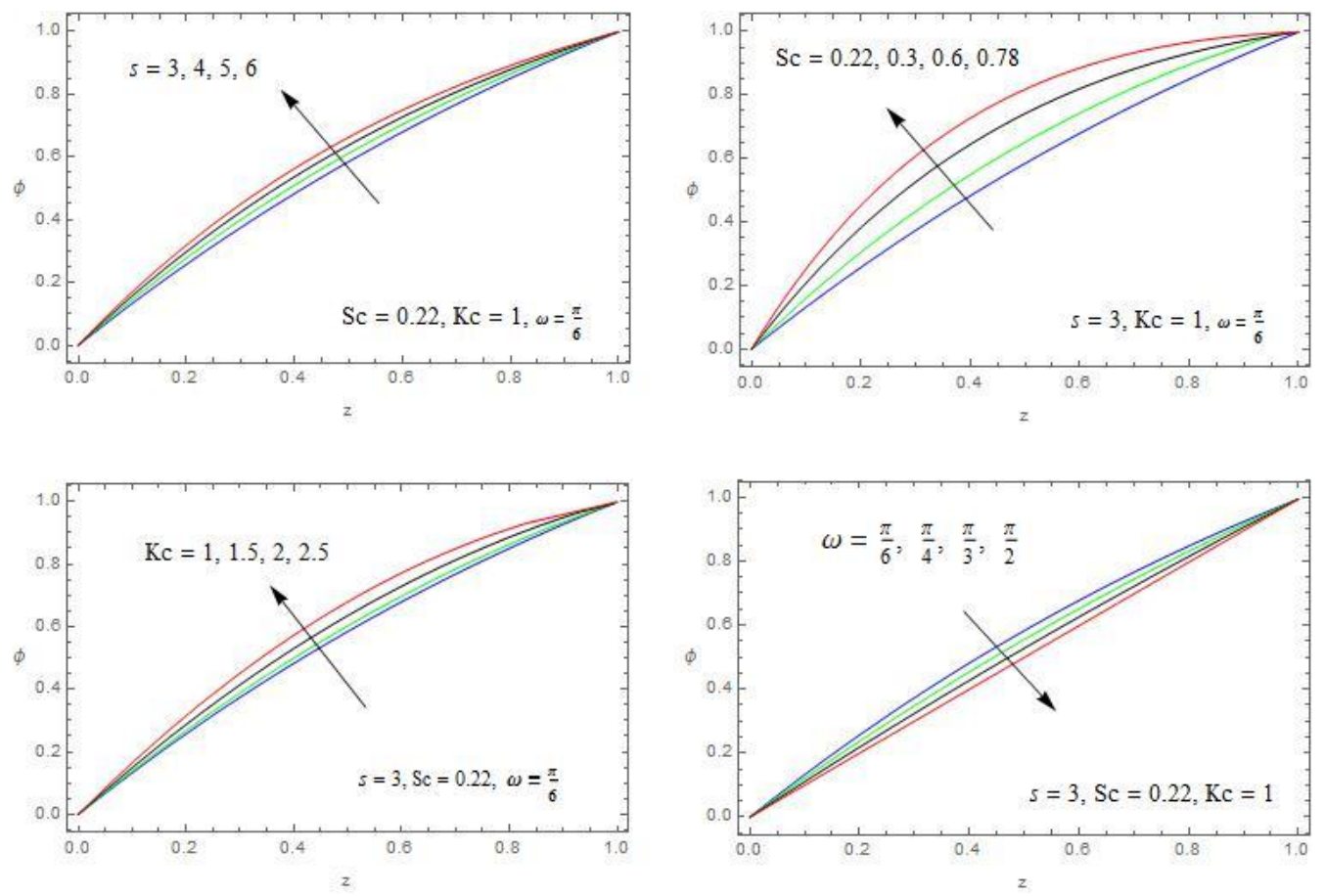

Fig. 7: The Concentration profiles against $s, \mathrm{Sc}, \mathrm{Kc}$ and $\omega$

Table 1: Shear stress

\begin{tabular}{|c|c|c|c|c|c|c|c|c|c|c|c|c|c|c|}
\hline$m$ & $\alpha$ & $M$ & $K$ & $\mathrm{Gr}$ & $\mathrm{Gm}$ & $s$ & $\operatorname{Pr}$ & $\mathrm{Sc}$ & $\mathrm{Kc}$ & $\gamma$ & $\omega$ & $\delta$ & $S h_{\mathrm{L}}$ & $S h_{\mathrm{R}}$ \\
\hline 1 & 1 & 0.5 & 0.1 & 1 & 2 & 1 & 0.71 & 0.22 & 1 & 0.5 & $\pi / 4$ & 0.5 & 0.554588 & 2.78025 \\
\hline 2 & & & & & & & & & & & & & 0.688599 & 2.66015 \\
\hline 3 & & & & & & & & & & & & & 0.799520 & 2.52001 \\
\hline & 2 & & & & & & & & & & & & 0.870114 & 2.57485 \\
\hline & 3 & & & & & & & & & & & & 1.267485 & 2.38545 \\
\hline & & 1 & & & & & & & & & & & 0.448955 & 2.64115 \\
\hline & & 1.5 & & & & & & & & & & & 0.414115 & 2.57485 \\
\hline & & & 0.2 & & & & & & & & & & 2.133622 & 2.78895 \\
\hline & & & 0.3 & & & & & & & & & & 3.241022 & 2.79999 \\
\hline & & & & 2 & & & & & & & & & 0.646625 & 2.97402 \\
\hline & & & & 3 & & & & & & & & & 0.700145 & 3.24452 \\
\hline & & & & & 3 & & & & & & & & 0.697440 & 3.00021 \\
\hline & & & & & 4 & & & & & & & & 0.737485 & 3.38554 \\
\hline & & & & & & 1.5 & & & & & & & 0.963662 & 3.00145 \\
\hline & & & & & & 2 & & & & & & & 1.518590 & 3.34521 \\
\hline & & & & & & & 3 & & & & & & 2.408595 & 2.64102 \\
\hline & & & & & & & 6 & & & & & & 2.807448 & 2.55201 \\
\hline & & & & & & & & 0.3 & & & & & 0.527401 & 2.73320 \\
\hline & & & & & & & & 0.6 & & & & & 0.429989 & 2.66201 \\
\hline & & & & & & & & & 1.5 & & & & 0.548500 & 2.75142 \\
\hline & & & & & & & & & 2 & & & & 0.545748 & 2.74156 \\
\hline & & & & & & & & & & 0.75 & & & 0.338744 & 2.39985 \\
\hline & & & & & & & & & & 1 & & & 0.240114 & 2.23325 \\
\hline & & & & & & & & & & & $\pi / 3$ & & 0.534748 & 2.67785 \\
\hline & & & & & & & & & & & $\pi / 2$ & & 0.918502 & 2.52214 \\
\hline & & & & & & & & & & & & 1 & 0.538023 & 2.73022 \\
\hline & & & & & & & & & & & & 1.5 & 0.526014 & 2.68899 \\
\hline
\end{tabular}


Table 2: Nusselt number

\begin{tabular}{|c|c|c|c|c|c|c|}
\hline $\mathbf{P r}$ & $\delta$ & $\boldsymbol{s}$ & $\boldsymbol{\omega}$ & $\boldsymbol{t}$ & $\mathbf{N u}_{\mathbf{L}}$ & $\mathbf{N u}_{\mathbf{R}}$ \\
\hline 0.71 & 1 & 5 & $\pi / 6$ & 0.2 & 2.02621 & 0.336515 \\
\hline 3 & & & & & 2.40242 & 0.193257 \\
\hline 7 & & & & & 2.93776 & 0.0825181 \\
\hline & 2 & & & & 2.12926 & 0.238023 \\
\hline & 3 & & & & 2.23981 & 0.135489 \\
\hline & & 6 & & & 2.26133 & 0.264963 \\
\hline & & 7 & & & 2.50735 & 0.204642 \\
\hline & & & $\pi / 4$ & & 2.02662 & 0.336292 \\
\hline & & & $\pi / 3$ & & 2.02680 & 0.336189 \\
\hline & & & & 0.4 & 1.78082 & 0.266138 \\
\hline & & & & 0.6 & 1.05827 & 0.124449 \\
\hline
\end{tabular}

Table 3: Sherwood number

\begin{tabular}{|c|c|c|c|c|c|c|}
\hline $\mathbf{S c}$ & $\mathbf{K c}$ & $\boldsymbol{s}$ & $\boldsymbol{\omega}$ & $\boldsymbol{t}$ & $\boldsymbol{S h _ { \mathbf { L } }}$ & $\boldsymbol{S h _ { \mathbf { R } }}$ \\
\hline 0.22 & 2 & 5 & $\pi / 6$ & 0.2 & 1.67297 & 0.515315 \\
\hline 0.3 & & & & & 1.98550 & 0.371924 \\
\hline 0.4 & & & & & 2.43060 & 0.214464 \\
\hline & 3 & & & & 1.68636 & 0.499586 \\
\hline & 4 & & & & 1.69990 & 0.483757 \\
\hline & & 6 & & & 1.82723 & 0.448285 \\
\hline & & 7 & & & 1.98830 & 0.388109 \\
\hline & & & $\pi / 4$ & & 1.67169 & 0.514006 \\
\hline & & & $\pi / 3$ & & 1.66895 & 0.511179 \\
\hline & & & & 0.4 & 1.66425 & 0.510852 \\
\hline & & & & 0.6 & 1.64897 & 0.504372 \\
\hline
\end{tabular}

Table 4. Comparison of the results

(Velocity component $u$ at $\mathrm{z}=0.2$ level and $\alpha=0, m=0$ )

\begin{tabular}{|c|c|c|c|c|}
\hline M & K & Gr & $\begin{array}{c}\text { Previous results } \\
\text { Falade et al. [24] }\end{array}$ & $\begin{array}{c}\text { Present } \\
\text { results }\end{array}$ \\
\hline 2 & 0.5 & 3 & 0.258856 & 0.258788 \\
\hline 3 & & & 0.201145 & 0.201014 \\
\hline 4 & & & 0.152448 & 0.152390 \\
\hline & 1 & & 0.366552 & 0.366444 \\
\hline & 1.5 & & 0.452252 & 0.452102 \\
\hline & & 4 & 0.455895 & 0.455752 \\
\hline & & 5 & 0.644782 & 0.644745 \\
\hline
\end{tabular}

\section{CONCLUSIONS}

We concluded that,

1. The resultant velocity enhances with increasing Hall parameter or second grade fluid parameter.

2. When increasing the intensity of the magnetic field, the resultant velocity retards throughout the flow field, where as it increases with increasing permeability parameter.

3. Also lifts the temperature, decreases the Nusselt number at the impassioned plate and increases it at the freezing wall.

4. The concentration reduces with increasing Schmidt number, whereas it enhances with increasing chemical reaction parameter or the frequency of oscillation.

5. Enlarges the skin friction on either plate with suction parameter.

\section{REFERENCES}

[1]. Makinde. O.D., P.Y. Mhone, Heat transfer to MHD oscillatory flow in a channel filled with porous medium, Romanian J. Phys., vol. 50, pp. 931-938, 2005.

[2]. Mehmood.A., A. Ali, The effect of slip condition on unsteady MHD oscillatory flow of a viscous fluid In a planer channel, Romanian J. Phys., vol. 52, pp. 85-91, 2007.

[3]. Chauchan. D.S., V. Kumar, Radiation effects on mixed convection flow and viscous heating in a vertical channel partially filled with a porous medium, Tamkang J. Sci. Eng., vol. 14, pp. 97-106, 2011.

[4]. Palani.G., I.A. Abbas, Free convection MHD flow with thermal radiation from impulsively started vertical plate, Non-linear Anal.: Model. Contr., vol. 14, pp. 7384, 2009.

[5]. Hussain.M., T. Hayat, S. Asghar, C. Fetecau, Oscillatory flows of second grade fluid in a porous space, Nonlinear Anal.: Real World Appl., vol. 11, pp. 2403-2414, 2010.

[6]. Umavathi. J.C., A. J Chamkha, A. Mateenand, AlMudhat in a horizontal composite porous medium channel, Non-linear Anal.:Model. Contr., vol. 14, pp. 397-415, 2009.

[7]. Jha. B.K., A.O. Ajibade, Free convective flow between vertical porous plates with periodic heat input, $Z$. Angew. Math. Mech. vol. 90 (3), pp. 185-193, 2010. DOI: http://dx.doi.org/10.1002/zamm.200900268.

[8]. Jha.B.K., A.O. Ajibade, Free convective flow of heat generating/absorbing fluid between vertical porous plates with periodic heat input, Int. Commun. Heat Mass Transfer, vol. 36, pp. 624-631, 2009.

[9]. Jha. B.K., A.O. Ajibade, Effect of viscous dissipation on natural convection flow between vertical parallel plates with time periodic boundary conditions, 
Commun. Nonlinear Sci.Numer. Simul., vol. 17, pp. 1576-1587, 2012.

[10]. Adesanya. S.O., O.D. Makinde, Heat transfer to magnetohydrodynamic non-Newtonian couple stress pulsatile flow between two parallel porous plates, $Z$. Naturforsch., vol. 67a, pp. 647-656, 2013.

[11]. Adesanya.S.O., J.A. Gbadeyan, A domian decomposition approach to steady visco-elastic fluid flow with slip through a planar channel, Int. $J$. Nonlinear Sci. vol. 9, pp. 86-94, 2010.

[12]. Adesanya. S.O., E.O. Oluwadare, J.A. Falade, O.D. Makinde, Hydromagnetic natural convection flow between vertical parallel plates with time-periodic boundary conditions, J.Magn. Magn. Mater., vol. 396, pp. 295-303, 2015.

[13]. Adesanya S.O., Free convective flow of heat generating fluid through a porous vertical channel with velocity slip and temperature jump, Ain Shams Eng. J., vol. 6 (3), pp. 1045-1052, 2015.

[14]. Adesanya. S.O., J.A. Falade, O.D. Makinde, Pulsating flow through vertical porous channel with viscous dissipation effect, UPB. Sci. Bull. Ser. D., vol. 77 (1), pp. 25-36, 2015.

[15]. Adesanya, S.O., J.A. Falade, Thermodynamics analysis of hydromagnetic third grade fluid flow through a channel filled with porous medium, Alexandria Eng. $J$. vol. 54, pp. 615-622, 2015.

[16]. Sivaraj.R., B. Rushi Kumar, Unsteady MHD dusty visco-elasticfluid Couette flow in an irregular channel with varying mass diffusion, Int. J. Heat Mass Transfer 55 (11) (2012) 3076-3089.

[17]. Prakash.J., B.R. Kumar, R. Sivaraj, Radiation and Dufour effects on unsteady MHD mixed convective flow in an accelerated vertical wavy plate with varying temperature and mass diffusion, Walailak J. Sci. Technol., vol. 11(11), pp. 939-954, 2014.

[18]. Sivaraj.J., A. Jasmine Benazir, Unsteady magnetohydrodynamic mixed convective oscillatory flow of Casson fluid in a porous asymmetric wavy channel, Spec. Top.Rev. Porous Media: Int. J., vol. 6 (3), pp. 267-281, 2015.

[19]. Jasmine Benazir.A., R. Sivaraj, O.D. Makinde, Unsteady magnetohydrodynamic Casson fluid flow over a vertical cone and flat plate with non-uniform heat source/sink, Int. J. Eng.Res. Afr., vol.21, pp. 6983, 2016.

[20]. Jasmine Benazir.A., R. Sivaraj, M.M. Rashidi, Comparison between Casson fluid flow in the presence of heat and mass transfer from a vertical cone and flat plate, J. Heat Transfer, vol. 138 (11), p. 112005, 2016.

[21]. Veera Krishna.M., B.V.Swarnalathamma and J. Prakash, "Heat and mass transfer on unsteady MHD Oscillatory flow of blood through porous arteriole, Applications of Fluid Dynamics, Lecture Notes in Mechanical Engineering, vol. XXII, pp. 207-224,
2018. DOI: https://doi.org/10.1007/978-981-10-53290_14.

[22]. M.Veera Krishna, G.Subba Reddy, A.J.Chamkha, "Hall effects on unsteady MHD oscillatory free convective flow of second grade fluid through porous medium between two vertical plates," Physics of Fluids, vol. 30, p. 023106, 2018. DOI: https://doi.org/10.1063/1.5010863

[23]. Veera Krishna.M, A.J.Chamkha, Hall effects on unsteady MHD flow of second grade fluid through porous medium with ramped wall temperature and ramped surface concentration, Physics of Fluids, vol. 30, p. $053101, \quad 2018$. DOI: https://doi.org/10.1063/1.5025542

[24]. Falade. J.A., Joel C. Ukaegbu, A.C. Egere, Samuel O. Adesanya, MHD oscillatory flow through a porous channel saturated with porous medium, Alexandria Engineering Journal, vol. 56, pp. 147-152, 2017.DOI: http://dx.doi.org/10.1016/j.aej.2016.09.016

[25]. Veera Krishna, M., Chamkha, A.J., Hall and ion slip effects on MHD rotating boundary layer flow of nanofluid past an infinite vertical plate embedded in a porous medium, Results in Physics, 15, 102652, DOI: https:// doi.org/ 10.1016/j.rinp.2019.102652

[26]. Veera Krishna, M., Swarnalathamma, B.V., Chamkha, A.J., Investigations of Soret, Joule and Hall effects on MHD rotating mixed convective flow past an infinite vertical porous plate, Journal of Ocean Engineering and Science, 4(3), pp. 263-275, 2019. DOI: https://doi.org/10.1016/j.joes.2019.05.002.

[27]. Veera Krishna.M., Chamkha, A.J., Hall effects on MHD Squeezing flow of a water based nano fluid between two parallel disks, Journal of Porous media, 22(2), pp. 209-223, 2019. DOI: https://doi.org/10.1615/JPorMedia.2018028721 